\title{
EXISTENCE OF POSITIVE SOLUTIONS FOR A CLASS OF THE $p$-LAPLACE EQUATIONS
}

\author{
YIN XI HUANG ${ }^{1}$
}

(Received 26 March 1992; revised 9 March 1993)

\begin{abstract}
We are concerned with the existence of solutions of

$$
-\Delta_{p} u=f(x, u)+h(x) \text { in } \Omega, \quad u=0 \text { on } \partial \Omega,
$$

where $\Delta_{p}$ is the $p$-Laplacian, $p \in(1, \infty)$, and $\Omega$ is a bounded smooth domain in $\mathbb{R}^{n}$.

For $h(x) \equiv \mathbf{0}$ and $f(x, u)$ satisfying proper asymptotic spectral conditions, existence of a unique positive solution is obtained by invoking the sub-supersolution technique and the spectral method. For $h(x) \not \equiv 0$, with assumptions on asymptotic behavior of $f(x, u)$ as $u \rightarrow \pm \infty$, an existence result is also proved.
\end{abstract}

\section{Introduction}

In this paper we investigate the existence of solutions for the Dirichlet problem of the following quasilinear elliptic equation:

$$
-\Delta_{p} u=f(x, u)+h(x) \quad \text { in } \Omega, \quad u=0 \text { on } \partial \Omega,
$$

where $\Omega$ is a bounded domain in $\mathbb{R}^{n}$ with smooth boundary $\partial \Omega, p \in(1, \infty), \Delta_{p} u=$ $\operatorname{div}\left(|\nabla u|^{p-2} \nabla u\right)$ is the $p$-Laplacian, $h(x) \in L^{\infty}$ and $f$ is to be specified below.

For $p \neq 2$, operator $\Delta_{p}$ has appeared in a variety of physical fields. For example, applications of $\Delta_{p}$ have been seen in fluid dynamics. The shear stress $\vec{\tau}$ and the velocity gradient $\nabla u$ of the fluid are related in the manner that $\vec{\tau}(x)=r(x)|\nabla u|^{p-2} \nabla u$, where $p=2$ (respectively, $p<2, p>2$ ) if the fluid is Newtonian (respectively, pseudoplastic, dilatant). Consequently the equation governing the motion of the fluid involves the $p$-Laplacian. The $p$-Laplacian also appears in the study of flow through porous media $(p=3 / 2)$, nonlinear elasticity $(p \geq 2)$ and glaciology $(p \in(1,4 / 3])$. We refer to Diaz [8] and Lions [14] for more background material and applications. For

\footnotetext{
${ }^{1}$ Department of Mathematical Sciences, Memphis State University, Memphis, TN 38152, U.S.A

(C) Australian Mathematical Society, 1994, Serial-fee code 0334-2700/94
} 
$f$ homogeneous in $u$, that is, $f(x, u)=f(x) u^{\sigma}(\sigma>0)$, existence and nonexistence results were obtained by many authors, see for example Azorero and Alonso [2], Diaz [8], Guedda and Veron [10], Lions [14], Otani [15] and Szulkin [19]. Variational methods were employed extensively when trying to find positive solutions. If $f^{+}(x)=$ $\max (f(x), 0) \not \equiv 0$, eigenvalue problem $-\Delta_{p} u=\lambda f(x)|u|^{p-2} u$ with homogeneous Dirichlet condition has been proved to possess a unique simple positive eigenvalue with positive eigenfunction. See Anane [1], Azorero and Alonso [2], and Otani and Teshima [16]. For the case where $f(x, \cdot)$ is of order $u^{p-1}$ but nonhomogeneous in $u$, Del Pino, Elgueta and Manasevich [6] studied the existence of solutions of (1.1) with $h \equiv 0$ for the one dimensional case, Boccardo, Drátbek and Kučera [3] considered the higher dimensional case with $h \not \equiv 0$. While for $p=2$, problem (1.1) has been studied extensively, see for example Brezis and Oswald [4], Costa and Gonçalves [5], Kazdan and Kramer [12] and references therein.

The objective of this paper is to extend some of the known results for the special case $p=2$ to the general quasilinear case. In particular, we will obtain the following result:

With $h(x) \equiv 0$, if $f(x, t)=f(t), \liminf _{t \rightarrow 0^{+}} f(t) / t^{p-1}=a_{0}, \limsup _{t \rightarrow \infty} f(t) / t^{p-1}$ $=a_{\infty}$, and

$$
0<a_{\infty}<\lambda_{1}<a_{0}
$$

where $\lambda_{1}$ is the smallest positive eigenvalue of $-\Delta_{p} u=\lambda|u|^{p-2} u$ with homogeneous Dirichlet condition, then problem (1.1) has a positive solution. Moreover, the solution is unique and condition $(*)$ is necessary if $f(t) / t^{p-1}$ is strictly decreasing. The method we employ follows the sub-supersolution procedure utilized in [5]. The construction of a sub-supersolution pair is made feasible by establishing some properties for indefinite eigenvalue problem for the $p$-Laplacian, which are of independent interest and extend the known results of Hess and Kato [11] for second order elliptic operators. For $h(x) \not \equiv 0$ we decompose $f(x, u)$ into

$$
f(x, u)=a_{+}(x)|u|^{p-2} u^{+}-a_{-}(x)|u|^{p-2} u^{-}+f_{1}(x, u),
$$

with $f_{1}(x, u)$ as the lower order term in $u$, and use topological degree theory to establish an existence result. We note that, due to the set-up of our problem, more general existence results are actually obtained.

The rest of the paper is organized as follows. In Section 2, we introduce the needed notations and prove several lemmas which provide the foundation for the construction of a sub-supersolution pair as well as for utilization of topological degree theory. In Section 3, the sub-supersolution pair is constructed and the existence of positive solution of (1.1) is obtained. Finally, Section 4 studies the case $h(x) \not \equiv 0$. 


\section{Preliminaries}

In this section we present some notations and results needed in the sequel. For the special case $p=2$, these results are well known, $c f$. Hess and Kato [11].

Let $\Omega$ be a smooth bounded domain in $\mathbb{R}^{n}$. We denote $\Delta_{p} u=\operatorname{div}\left(|\nabla u|^{p-2} \nabla u\right)$ for $u$ in $W^{1, p}(\Omega)$ with $p \in(1, \infty)$. By Theorems 2.2.1 and 2.2.3 of Lions [14], the problem

$$
-\Delta_{p} u=f(x) \quad \text { in } \Omega, \quad u=0 \text { on } \partial \Omega,
$$

has a unique solution $u \in W_{0}^{1, p}(\Omega)$ for any $f \in L^{q}(1 / p+1 / q=1)$. Regularity theorems (see Tolksdorf [20, page 806] and Di Benedetto [7]) further show that if $f \in L^{\infty}(\Omega)$, then $u \in C^{1+\alpha}(\bar{\Omega})$ for some $0<\alpha<1$. By uniqueness of the solution of (2.1), the inverse of $-\Delta_{p}$ exists and is henceforth denoted by $R: L^{\infty} \rightarrow L^{\infty}$. We first have

LEMMA 2.1. $R$ is compact, continuous and strictly positive, that is, for $f \geq 0, f \neq 0$, $R f>0$.

PROOF. Compactness follows from the fact that $C^{1+\alpha}(\bar{\Omega})$ is embedded in $L^{\infty}(\Omega)$ compactly and densely.

Let $u_{k}=R\left(f_{k}\right), f_{k} \rightarrow f_{0}$ in $L^{\infty}$. By compactness, for some $u_{0} \in L^{\infty} \cap W_{0}^{1, p}(\Omega)$, $u_{k} \rightarrow u_{0}$ in $L^{\infty}$, and $u_{k} \rightarrow u_{0}$ in $W_{0}^{1, p}(\Omega)$ weakly. For this $f_{0}$, there exists a $\tilde{u} \in L^{\infty}$ such that $\tilde{u}=R\left(f_{0}\right)$. It then follows from $u_{k}=R\left(f_{k}\right)$ and $\tilde{u}=R\left(f_{0}\right)$ that

$$
\begin{aligned}
J\left(u_{k}, \tilde{u}\right) & :=\int\left(\left|\nabla u_{k}\right|^{p-2} \nabla u_{k}-|\nabla \tilde{u}|^{p-2} \nabla \tilde{u}\right) \nabla\left(u_{k}-\tilde{u}\right) \\
& =\int\left(f_{k}-f_{0}\right)\left(u_{k}-\tilde{u}\right) .
\end{aligned}
$$

Note that for any vectors $a$ and $b$,

$$
|a-b|^{p} \leq c \cdot\left\{\left(|a|^{p-2} a-|b|^{p-2} b\right) \cdot(a-b)\right\}^{t / 2} \cdot\left(|a|^{p}+|b|^{p}\right)^{1-t / 2},
$$

where $t=\min \{p, 2\}$ (see Lindqvist [13], Appendix). Thus by Hölder's inequality we conclude

$$
\left\|\nabla u_{k}-\nabla \tilde{u}\right\|_{p}^{p} \leq c \cdot\left(J\left(u_{k}, \tilde{u}\right)\right)^{t / 2} \cdot\left(\left\|\nabla u_{k}\right\|_{p}^{p}+\|\nabla \tilde{u}\|_{p}^{p}\right)^{1-t / 2} .
$$

Consequently $u_{k} \rightarrow \tilde{u}$ in $W_{0}^{1, p}(\Omega)$. Thus $\tilde{u}=u_{0}$, and this implies continuity of $R$.

Let $u=R f$, then $-\Delta_{p} u=f$. Multiplying this equation by $u^{-}:=u^{+}-u$ and integrating show that $u^{-}=0$ on the set $(f(x) \neq 0)$ and $u^{-}=$const. on the set $(f(x)=0)$. We conclude from continuity of $u^{-}$that $u^{-}=0$, that is, $u \geq 0$. It then follows from Lemma 2 of Otani and Teshima [16] that $u>0$. This concludes the proof. 
REMARK 2.1. The above proof actually implies that, if $f_{k} \rightarrow f_{0}$ in $L^{q}\left(\frac{1}{p}+\frac{1}{q}=1\right)$, and $u_{k}:=R\left(f_{k}\right) \rightarrow$ (some) $u_{0} \in W_{0}^{1, p}(\Omega)$ weakly in $W_{0}^{1, p}(\Omega)$, then $u_{0}=R\left(f_{0}\right)$.

From now on we denote $r=n / p$ if $p \leq n$, and $r=1$ if $p>n$. Let $m(x) \in L^{r+\varepsilon}(\Omega)$ for some $\varepsilon>0, m^{+}(x)=\max (m(x), 0) \not \equiv 0$. Then the following is well known (see Anane [1], Azorero and Alonso [2], and Otani and Teshima [16]):

PROPOSITION 2.2. The eigenvalue problem

$$
-\Delta_{p} u=\lambda m(x)|u|^{p-2} \quad \text { in } \Omega, \quad u=0 \text { on } \partial \Omega
$$

has a unique positive eigenvalue $\lambda_{1}(m)$ which is simple and has an associated positive eigenfunction $\varphi \in W_{0}^{1, p}(\Omega) . \quad \lambda_{1}(m)$ is characterized by the following variational formula

$$
\lambda_{1}(m)=\inf \left\{\int|\nabla u|^{p}: \int m|u|^{p}=1, u \in W_{0}^{1, p}(\Omega)\right\} .
$$

Moreover, if $m \in L^{\infty}$, then $\partial \varphi / \partial \nu<0$ on $\partial \Omega$, where $v$ denotes the outward normal of $\partial \Omega$.

Next we use the variational characteristic of $\lambda_{1}(m)$ to establish

LEMMA 2.3. Let $\lambda_{1}(m)$ be as given in Proposition 2.2 and $m(x) \in L^{\infty}(\Omega)$. Then

(i) $\lambda_{1}$ is decreasing in $m$, that is, $m_{1}>m_{2}$ with $m_{2}^{+} \not \equiv 0$ implies that $\lambda_{1}\left(m_{1}\right)<$ $\lambda_{1}\left(m_{2}\right)$.

(ii) If $m_{k} \rightarrow m_{0}$ in $L^{\infty}$ with $m_{0}^{+} \not \equiv 0$, then $\lambda_{1}\left(m_{k}\right) \rightarrow \lambda_{1}\left(m_{0}\right)$.

PROOF. (i). For $u \in W_{0}^{1, p}(\Omega)$ with $\int m_{2}|u|^{p}=1$, we have $a:=\int m_{1}|u|^{p} \geq 1$. We then derive that $\int|\nabla u|^{p} \geq a \lambda_{1}\left(m_{1}\right)$, thus $\lambda_{1}\left(m_{1}\right) \leq \lambda_{1}\left(m_{2}\right)$.

Suppose $\lambda_{1}\left(m_{1}\right)=\lambda_{1}\left(m_{2}\right)$. Let $u_{1}, u_{2}$ be the positive eigenfunctions associated with $\lambda_{1}\left(m_{1}\right), \lambda_{1}\left(m_{2}\right)$ respectively and set

$$
I\left(u_{1}, u_{2}\right):=\int\left(-\Delta_{p} u_{1}\right) \frac{u_{1}^{p}-u_{2}^{p}}{u_{1}^{p-1}}-\int\left(-\Delta_{p} u_{2}\right) \frac{u_{1}^{p}-u_{2}^{p}}{u_{2}^{p-1}} .
$$

We note that such $I\left(u_{1}, u_{2}\right)$ is well defined since both $\partial u_{1} / \partial \nu, \partial u_{2} / \partial v<0$ on $\partial \Omega(c f$. Anane [1]). Calculation shows

$$
I\left(u_{1}, u_{2}\right)=\int \lambda_{1}\left(m_{1}\right)\left(m_{1}-m_{2}\right)\left(u_{1}^{p}-u_{2}^{p}\right) .
$$

By choosing $u_{1}-u_{2}<0$ in $\Omega$ we have $I\left(u_{1}, u_{2}\right) \leq 0$. It then follows from Proposition 1 of Anane [1] that $u_{1}=k u_{2}$ for some constant $k$, a contradiction. Hence $\lambda_{1}\left(m_{1}\right)<\lambda_{1}\left(m_{2}\right)$. 
(ii). We first claim that $\lambda_{1}\left(m_{k}\right)$ is bounded. Indeed, let $u_{0}$ be the positive eigenfunction associated with $\lambda_{1}\left(m_{0}\right)$ satisfying $\int m_{0}\left|u_{0}\right|^{p}=1$. For $k$ sufficiently large, we have $1 / 2 \leq \int m_{k}\left|u_{0}\right|^{p} \leq 3 / 2$. We thus conclude that $\lambda_{1}\left(m_{k}\right) \leq\left(\int\left|\nabla u_{0}\right|^{p}\right) /\left(\int m_{k}\left|u_{0}\right|^{p}\right)$ is bounded.

Now, since $\int\left|\nabla u_{k}\right|^{p}$ is bounded, where $u_{k}$ is the positive eigenfunction associated with $\lambda_{1}\left(m_{k}\right)$ satisfying $\int m_{k}\left|u_{k}\right|^{p}=1$, we may assume, taking a subsequence if necessary, that for some $\tilde{u} \in W_{0}^{1, p}(\Omega), u_{k} \rightarrow \tilde{u}$ weakly in $W_{0}^{1, p}(\Omega), u_{k} \rightarrow \tilde{u}$ in $L^{p}$ if $p<n$ and in Cif $p \geq n$, and $\lambda_{1}\left(m_{k}\right) \rightarrow \tilde{\lambda}$. It then follows that $\int m_{k}\left|u_{k}\right|^{p} \rightarrow \int m_{0}|\tilde{u}|^{p}$. Since $u_{k}$ satisfies

$$
-\Delta_{p} u_{k}=\lambda_{1}\left(m_{k}\right) m_{k} u_{k}^{p-1} \quad \text { in } \Omega, \quad u_{k}=0 \text { on } \partial \Omega,
$$

we let

$$
J\left(u_{k}, u_{l}\right):=\int\left(\left|\nabla u_{k}\right|^{p-2} \nabla u_{k}-\left|\nabla u_{l}\right|^{p-2} \nabla u_{l}\right) \nabla\left(u_{k}-u_{l}\right),
$$

then, as in the proof of Lemma 2.1, we have

$$
\left\|\nabla u_{k}-\nabla u_{l}\right\|_{p}^{p} \leq c \cdot\left(J\left(u_{k}, u_{l}\right)\right)^{t / 2} \cdot\left(\left\|\nabla u_{k}\right\|_{p}^{p}+\left\|\nabla u_{l}\right\|_{p}^{p}\right)^{1-t / 2},
$$

where $t=\min \{p, 2\}$. We then derive that $u_{k}$ is a Cauchy sequence in $W_{0}^{1, p}(\Omega)$ and hence $u_{k} \rightarrow \tilde{u}$ in $W_{0}^{1, p}(\Omega)$.

We now claim that $\tilde{\lambda}=\lambda_{1}\left(m_{0}\right)$. Assume, on the contrary, that $\tilde{\lambda}-\lambda_{1}\left(m_{0}\right)=\varepsilon>0$. Since $m_{k} \rightarrow m_{0}$ in $L^{\infty}$, we have, for $k$ sufficiently large,

$$
\int\left|\nabla u_{0}\right|^{p} / \int m_{k}\left|u_{o}\right|^{p}-\lambda_{1}\left(m_{0}\right)<\varepsilon / 4
$$

and

$$
\left|\lambda_{1}\left(m_{k}\right)-\tilde{\lambda}\right|<\varepsilon / 4
$$

We then obtain that

$$
\begin{aligned}
0<\int\left|\nabla u_{0}\right|^{p} / \int m_{k}\left|u_{o}\right|^{p} & <\lambda_{1}\left(m_{0}\right)+\varepsilon / 4 \\
& =\tilde{\lambda}-3 \varepsilon / 4<\lambda_{1}\left(m_{k}\right)-\varepsilon / 2,
\end{aligned}
$$

which contradicts the variational characteristic of $\lambda_{1}\left(m_{k}\right)$. Consequently we have $\tilde{\lambda}=\lambda_{1}\left(m_{0}\right)$. The proof is complete.

REMARK 2.2. It follows from the above proof that $\tilde{u}=u_{0}$, which implies that the positive eigenfunction $u$ is continuous in $W_{0}^{1, p}(\Omega)$ as a function of $m$.

To establish next lemma some definitions are needed. 
DEFINITION 2.1. Let $K$ be a closed convex subset of a Banach space $X$. Suppose $A: K \rightarrow X^{*}$ is bounded.

(i) $A$ is called a pseudomonotone operator if it satisfies the following condition: if $x_{k} \rightarrow x$ weakly in $X$ and $\lim \sup _{k \rightarrow \infty}\left\langle A\left(x_{k}\right), x_{k}-x\right\rangle \leq 0$, then

$$
\langle A(x), x-y\rangle \leq \liminf _{k \rightarrow \infty}\left\langle A\left(x_{k}\right), x_{k}-y\right\rangle
$$

for all $y \in X$.

(ii) $A$ is coercive if $\langle A(x), x\rangle /\|x\|_{X} \rightarrow \infty$ as $\|x\|_{X} \rightarrow \infty$.

The following lemma is standard and can be found in, for example, Lions [14].

LEMMA 2.4. Assume that $A$ is pseudomonotone and coercive, $X$ is a reflexive Banach space and $K$ is a closed convex subset of $X$. Then, for any $f \in X^{*}$, there exists an $x \in K$ such that for all $y \in K$,

$$
\langle A(x)-f, y-x\rangle \geq 0 .
$$

Now we are ready to introduce

LEMMA 2.5. Let $\lambda \in\left(0, \lambda_{1}(m)\right)$. Then for any $h \in W^{1, r}, h \geq 0$ in $\Omega$ and $h \not \equiv 0$, there exists a nonnegative solution $u \in W_{0}^{1, p}(\Omega)$ of

$$
-\Delta_{p} u=\lambda m(x)|u|^{p-2} u+h(x) \quad \text { in } \Omega, \quad u=0 \text { on } \partial \Omega .
$$

If, in addition, $m, h \in L^{\infty}$, then $u>0$ in $\Omega, \partial u / \partial \nu<0$ on $\partial \Omega$ and $u$ is unique.

PROOF. We first prove the existence part. We introduce an operator $A=A_{1}+A_{2}$ from $W_{0}^{1, p}(\Omega)$ to $\left(W_{0}^{1, p}(\Omega)\right)^{\prime}$ by

$$
\begin{aligned}
(A u, v) & =\left(A_{1} u, v\right)+\left(A_{2} u, v\right) \\
& =\int|\nabla u|^{p-2} \nabla u \nabla v-\lambda \int m|u|^{p-2} u v .
\end{aligned}
$$

Obviously $A$ is bounded. Next we verify that $A$ is pseudomonotone and coercive.

Indeed, for some $\alpha \in(0,1), \alpha \lambda_{1}(m)=\lambda$, we have, for $u \in W_{0}^{1, p}(\Omega)$,

$$
\begin{aligned}
\int|\nabla u|^{p} & =(1-\alpha) \int|\nabla u|^{p}+\alpha \int|\nabla u|^{p} \\
& \geq(1-\alpha) \int|\nabla u|^{p}+\alpha \lambda_{1}(m) \int m|u|^{p} \\
& =(1-\alpha) \int|\nabla u|^{p}+\lambda \int m|u|^{p} .
\end{aligned}
$$


Hence we deduce that

$$
(A u, u) \geq(1-\alpha)\|u\|_{W^{1 . p}}^{p},
$$

which implies coerciveness of $A$.

Note that $A_{1}$ is pseudomonotone since it is monotone (see Lions [14]) while $A_{2}$ is continuous. Thus $A=A_{1}+A_{2}$ is pseudomonotone.

Now we apply Lemma 2.4 to the sets $X=K=W_{0}^{1, p}(\Omega)$ to conclude the existence of a solution $u$ to (2.4).

To prove nonnegativity of the solution $u$, we proceed as follows. Since $u \in$ $W_{0}^{1, p}(\Omega)$, we have $u^{-} \in W_{0}^{1, p}(\Omega)$ (see Gilbarg and Trudinger [9]). Multiplying (2.4) by $u^{-}$and integrating by parts, we obtain

$$
-\int\left|\nabla u^{-}\right|^{p}+\lambda \int m\left|u^{-}\right|^{p}=\int h u^{-} .
$$

Since $\lambda<\lambda_{1}(m)$, the left-hand side of (2.5) is negative if $u^{-} \not \equiv 0$ while the right-hand side is nonnegative, a contradiction. We thus conclude that $u^{-} \equiv 0$, that is, $u \geq 0$.

Now, assume $m, h \in L^{\infty}$. We write (2.4) as

$$
-\Delta_{p} u+\lambda m^{-}|u|^{p-2} u=\lambda m^{+}|u|^{p-2} u+h(x) .
$$

$u>0$ in $\Omega$ and $\partial u / \partial v<0$ on $\partial \Omega$ then follow from Lemma 4 of Otani and Teshima [16].

Next, suppose $v>0$ is another solution. As in the proof of Lemma 2.3, we let

$$
I(u, v)=\int\left(-\Delta_{p} u\right) \frac{u^{p}-v^{p}}{u^{p-1}}-\int\left(-\Delta_{p} v\right) \frac{u^{p}-v^{p}}{v^{p-1}} .
$$

$I(u, v)$ is well defined since $\partial u / \partial v, \partial v / \partial v<0$ on $\partial \Omega$. If $u \not \equiv v$, then

$$
I(u, v)=\int \frac{h\left(u^{p}-v^{p}\right)\left(v^{p-1}-u^{p-1}\right)}{u^{p-1} v^{p-1}} \leq 0 .
$$

Again Proposition 1 of Anane [1] implies that $u=k v$ for some constant $k$. We thus conclude that $u \equiv v$. This completes the proof.

For general problem

$$
-\Delta_{p} u=f(x, u) \quad \text { in } \Omega, \quad u=0 \text { on } \partial \Omega,
$$

we introduce

DEFINITION 2.2. A function $\bar{u} \in W^{1, p}(\Omega)$ is said to be a supersolution of (2.6) if

$$
\int|\nabla \bar{u}|^{p-2} \nabla \bar{u} \nabla \varphi \geq \int f(x, \bar{u}) \varphi
$$

for all $\varphi \in C_{0}^{\infty}(\Omega), \varphi \geq 0$. Subsolution is defined accordingly. 


\section{Existence results}

To obtain the existence of a positive solution of

$$
-\Delta_{p} u=f(x, u) \quad \text { in } \Omega, \quad u=0 \text { on } \partial \Omega,
$$

we employ sub-supersolution technique, following Costa and Gonçalves [5] in principle. We postulate the following conditions on $f(x, u)$ throughout this section.

(H1) $f$ satisfies the Caratheodory condition, that is, $f$ is measurable with respect to $x$ for all $u \in \mathbb{R}$ and continuous with respect to $u$ for almost all $x \in \Omega$. Assume further that for some $\varepsilon>0$, and for any $K>0$,

$$
\sup _{0 \leq u \leq K}|f(x, u)| \in L^{r+\varepsilon} \text {. }
$$

(H2) The asymptotic limits

$$
a_{0}(x)=\liminf _{u \rightarrow 0^{+}} \frac{f(x, u)}{u^{p-1}}, \quad a_{\infty}(x)=\limsup _{u \rightarrow \infty} \frac{f(x, u)}{u^{p-1}}
$$

exist uniformly for a.e. $x \in \Omega$. Moreover, they satisfy that $a_{\infty} \in L^{\infty}, a_{\infty}^{+} \not \equiv 0$, and either $\infty>a_{0}(x)>c_{0}>0$ or $a_{0}(x) \equiv \infty$, and

$$
\lambda_{1}\left(a_{0}\right)<1<\lambda_{1}\left(a_{\infty}\right) .
$$

REMARK 3.1. For the case $a_{0}(x) \equiv+\infty$, we take $\lambda_{1}\left(a_{0}\right)=0$ in (3.4). If $a_{0}, a_{\infty}$ are constants, then (3.4) is equivalent to $0<a_{\infty}<\lambda_{1}(1)<a_{0}$, a condition used frequently for the case $p=2$, see for example Brezis and Oswald [4] and references therein.

We first construct a pair of sub-supersolution.

THEOREM 3.1. Assume (H1) and (H2) hold. Then there exists a positive supersolution $\bar{u} \in W_{0}^{1, p}(\Omega)$ of (3.1) such that $\partial \bar{u} / \partial v<0$ on $\partial \Omega$.

PROOF. We choose $\varepsilon>0$ small such that $\lambda_{1}\left(a_{\infty}+\varepsilon\right)>1$. This is possible by Lemma 2.3. By virtue of the definition of $a_{\infty}$, we can find a function $b_{\varepsilon}(x) \in W^{1, r} \cap L^{\infty}$ with $b_{\varepsilon} \geq 1$ in $\bar{\Omega}$ such that

$$
f(x, u) \leq\left(a_{\infty}+\varepsilon\right) u^{p-1}+b_{\varepsilon}
$$

for $u \geq 0$. Now we consider the following problem

$$
-\Delta_{p} u=\left(a_{\infty}+\varepsilon\right)|u|^{p-2} u+b_{\varepsilon} \quad \text { in } \Omega, \quad u=0 \text { on } \partial \Omega .
$$

Lemma 2.5 implies that (3.5) has a positive solution $u_{\varepsilon} \in W_{0}^{1, p}(\Omega)$ with $\partial u_{\varepsilon} / \partial \nu<0$ on $\partial \Omega$. We then choose $\vec{u}=u_{\varepsilon}$ to conclude the proof. 
THEOREM 3.2. Under the same conditions, there exists a positive subsolution $\underline{u} \in$ $W_{0}^{1, p}(\Omega)$ of (3.1) such that $\underline{u} \leq \bar{u}$.

PROOF. (i). For the case $a_{0}(x) \in L^{\infty}$, we again choose an $\varepsilon>0$ so small that $a_{0}-\varepsilon>0$ and $\lambda_{1}\left(a_{0}-\varepsilon\right)<1$. We further take $K>0$ small such that

$$
f(x, u) \geq\left(a_{0}-\varepsilon\right) u^{p-1}
$$

for all $0 \leq u \leq K$. Let $v_{1}$ be a positive eigenfunction of the following eigenvalue problem

$$
-\Delta_{p} u=\lambda_{1}\left(a_{0}-\varepsilon\right)\left(a_{0}-\varepsilon\right)|u|^{p-2} u \quad \text { in } \Omega, \quad u=0 \text { on } \partial \Omega .
$$

We can further assume that $v_{1} \leq K$. It then follows that

$$
\begin{aligned}
-\Delta_{p} v_{1} & =\lambda_{1}\left(a_{0}-\varepsilon\right)\left(a_{0}-\varepsilon\right) v_{1}^{p-1} \\
& \leq \lambda_{1}\left(a_{0}-\varepsilon\right) f\left(x, v_{1}\right) \leq f\left(x, v_{1}\right)
\end{aligned}
$$

since $\lambda_{1}\left(a_{0}-\varepsilon\right)<1$. We thus conclude that $v_{1}$ is a subsolution of (3.1). Since $\partial \bar{u} / \partial \nu<0$ and $\partial v_{1} / \partial \nu<0$ on $\partial \Omega$, we can choose $\alpha \in(0,1)$ small such that $\alpha v_{1} \leq \bar{u}$ in $\Omega$. Then $\underline{u}=\alpha v_{1}$ is as required.

(ii). For the case $a_{0}(x) \equiv+\infty$, replace in the above proof $a_{0}-\varepsilon$ by a large $M>0$. Then the same conclusion can be deduced. The proof is complete.

The next theorem, originally due to Kazdan and Kramer [12] for $p=2$, guarantees the existence of solutions for our problem.

THEOREM 3.3. Let $\underline{u}, \bar{u} \in W_{0}^{1, p} \cap L^{\infty}$ be a pair of sub-supersolution of (3.1). If $\underline{u} \leq \bar{u}$, then (3.1) has a solution $u \in W_{0}^{1, p}(\Omega)$ such that $\underline{u} \leq u \leq \bar{u}$ if $(H 1)$ is assumed.

REMARK 3.2. The proof we give below follows the same arguments as that of Kazdan and Kramer [12] and Costa and Gonçalves [5] for $p=2$, but we require and obtain weaker regularity. In fact, as indicated by Tolksdorf [20], one cannot expect $C^{2}$ regularity if $p<n$ and $p \neq 2$. A different proof for the case $p=2$ was given in Sattinger [17], where it was assumed that $f(x, u)+k u$ is nondecreasing in $u$ for some $k \geq 0$.

PROOF. For the given sub-supersolution pair $\underline{u} \leq \bar{u}$ in $L^{\infty}$, we define an operator $T$ by

$$
T u= \begin{cases}\underline{u}, & \text { if } u(x) \leq \underline{u}(x) \\ u, & \text { if } \underline{u}(x) \leq \vec{u}(x) \leq \vec{u}(x) \\ \bar{u}, & \text { if } u(x) \geq \bar{u}(x)\end{cases}
$$


and consider the problem

$$
-\Delta_{p} u+|u|^{p-2} u=f(x, T u)+|T u|^{p-2} T u \quad \text { in } \Omega, \quad u=0 \text { on } \partial \Omega .
$$

We first claim that problem (3.1) together with condition $\underline{u} \leq u \leq \bar{u}$ is equivalent to problem (3.6).

Obviously, solution $u$ of (3.1) satisfying $\underline{u} \leq u \leq \bar{u}$ also solves (3.6). On the other hand, let $u$ be a solution of (3.6) and denote $\tilde{u}=u-\bar{u}$. We will show that $\tilde{u} \leq 0$. Since $\bar{u}$ is a supersolution of (3.1), we have

$$
\begin{aligned}
\int\left(|\nabla \bar{u}|^{p-2} \nabla \bar{u}-|\nabla u|^{p-2} \nabla u\right) \nabla \varphi & \\
& \geq \int\left(f(x, \bar{u})-f(x, T u)-|T u|^{p-2} T u+|u|^{p-2} u\right) \varphi
\end{aligned}
$$

for all $\varphi \in W_{0}^{1, p}(\Omega), \varphi \geq 0$. If $\tilde{u}^{+} \not \equiv 0$, taking $\varphi=\tilde{u}^{+}$in the above inequality yields

$$
0>\int\left(|\nabla \bar{u}|^{p-2} \nabla \bar{u}-|\nabla u|^{p-2} \nabla u\right) \nabla \tilde{u}^{+} \geq 0,
$$

a contradiction. Hence $u \leq \bar{u}$. Analogously we can prove $\underline{u} \leq u$. It then follows that $\underline{u} \leq u \leq \bar{u}$ and consequently $u$ solves (3.1).

Now we consider the solvability of a family of problems

$$
-\Delta_{p} u+|u|^{p-2} u=t\left(f(x, T u)+|T u|^{p-2} T u\right) \quad \text { in } \Omega, \quad u=0 \text { on } \partial \Omega,
$$

for $t \in[0,1]$. It is easy to verify that solution of (3.7) is a fixed point of the following operator

$$
R K_{t}(u):=R\left(t\left(f(x, T u)+|T u|^{p-2} T u\right)-|u|^{p-2} u\right),
$$

where $R$ is the inverse of $-\Delta_{p}$ defined in Section 2. We claim that $R K_{t}$ is compact from $L^{\infty}$ to $L^{\infty}$ for all $t \in[0,1]$. Indeed we need only to verify that $R K$, is bounded since $R$ is compact (see Lemma 2.1). Let $v=R K_{t}(u)$, that is,

$$
-\Delta_{p} v=t\left(f(x, T u)+|T u|^{p-2} T u\right)-|u|^{p-2} u .
$$

For $p>n, L^{\infty}$ boundedness follows from the Sobolev embedding theorem since $v \in W_{0}^{1, p}(\Omega)$. For $p<n$, since for some $M>0, M \geq\|\underline{u}\|_{\infty},\|\bar{u}\|_{\infty}$, then by (H1), the norm of $f(x, T u)$ in $L^{r+\varepsilon}$ is independent of $u$, thus Theorem 1 of Serrin [18] implies that $\|v\|_{\infty} \leq M_{1}$, where $M_{1}$ is independent of $u$ and $t \in[0,1]$. For $p=n$, Theorem 2 of Serrin [18] implies the same.

The above analysis also shows that there exists a constant $M_{0}>0$, such that for all $t \in[0,1], R K_{t}$ has no fixed points outside the ball $B:=B_{M_{0}}$ in $L^{\infty}$, where $B_{M_{0}}$ is centered at the origin with radius $M_{0}$. Thus we conclude

$$
\operatorname{deg}\left(I-R K_{1}, B, 0\right)=\operatorname{deg}\left(I-R K_{0}, B, 0\right) .
$$


If $I-t R K_{0}$ has a zero point $u_{0}$, then $-\Delta_{p} u_{0}=-t^{p-1}\left|u_{0}\right|^{p-2} u_{0}$. Multiplying both sides of the equation by $u_{0}$ and integrating by parts we obtain

$$
0 \leq \int\left|\nabla u_{0}\right|^{p}=-t^{p-1} \int\left|u_{0}\right|^{p} \leq 0
$$

which implies $u_{0}=0$ if $t \neq 0$. This shows that $I-t R K_{0}$ is homotopic to $I$. It then follows that

$$
\operatorname{deg}\left(I-R K_{1}, B, 0\right)=\operatorname{deg}\left(I-R K_{0}, B, 0\right)=1,
$$

that is, there exists a solution to $u \in B$ of (3.7) with $t=1$. Consequently we obtain a solution $u$ of (3.1) with $\underline{u} \leq u \leq \bar{u}$. This ends the proof.

Now, combining Theorems 3.1, 3.2 and 3.3 we have

THEOREM 3.4. Assume that $(H I)$ and $(H 2)$ hold. Then there exists a positive solution $u \in W_{0}^{1, p}(\Omega) \cap L^{\infty}(\Omega)$ of (3.1). If, in addition, $f(x, t) / t^{p-l}$ is decreasing for $t \in(0, \infty)$, then the solution is unique in $L^{\infty}(\Omega)$.

PROOF. We need only verify the uniqueness part.

Let $v>0$ be another solution. Proposition 1 of [1] is not readily applicable here since we do not know whether $\partial v / \partial n<0$ on $\partial \Omega$ or not. Instead we adapt a device due to Lindqvist [13]. Let

$$
u_{\varepsilon}=u+\varepsilon, \quad v_{\varepsilon}=v+\varepsilon,
$$

for $\varepsilon>0$, and choose test function $\left(u_{\varepsilon}^{p}-v_{\varepsilon}^{p}\right) / u_{\varepsilon}^{p-1}$ for equation (3.1), and ( $u_{\varepsilon}^{p}-$ $\left.v_{\varepsilon}^{p}\right) / v_{\varepsilon}^{p-1}$ for (3.1) with $u$ replaced by $v$, respectively. Multiplying (3.1) and the corresponding equation for $v$ by the test functions and subtracting the resultant equations, we obtain

$$
\int\left(\left(-\Delta_{p} u\right) \frac{u_{\varepsilon}^{p}-v_{\varepsilon}^{p}}{u_{\varepsilon}^{p-1}}-\left(-\Delta_{p} v\right) \frac{u_{\varepsilon}^{p}-v_{\varepsilon}^{p}}{v_{\varepsilon}^{p-1}}\right)=\int\left(\frac{f(x, u)}{u_{\varepsilon}^{p-1}}-\frac{f(x, v)}{v_{\varepsilon}^{p-1}}\right)\left(u_{\varepsilon}^{p}-v_{\varepsilon}^{p}\right) .
$$

It is apparent that, by our assumption,

$$
\lim _{\varepsilon \rightarrow 0} \int\left(\frac{f(x, u)}{u_{\varepsilon}^{p-1}}-\frac{f(x, v)}{v_{\varepsilon}^{p-1}}\right)\left(u_{\varepsilon}^{p}-v_{\varepsilon}^{p}\right)=\int\left(\frac{f(x, u)}{u^{p-1}}-\frac{f(x, v)}{v^{p-1}}\right)\left(u^{p}-v^{p}\right) \leq 0 .
$$

Then the same arguments as that in the proof of Lemma 1 of [13] imply that $u=k v$ for some $k>0$. It is easy to see that $k=1$, that is, $u=v$. The proof is complete.

Finally we state a theorem which says that, in essence, $(\mathrm{H} 2)$ is necessary. 
THEOREM 3.5. Assume that, for all $x \in \bar{\Omega}, f(x, t) / t^{p-1}$ is strictly decreasing for $t \in(0, \infty)$ and $a_{0} \in L^{\infty}$. If (3.1) has a positive solution $u$, then

$$
\lambda_{1}\left(a_{0}\right)<1<\lambda_{1}\left(a_{\infty}\right) .
$$

PROOF. By monotonicity of $f(x, t) / t^{p-1}$, we have

$$
a_{\infty}(x)<f(x, t) / t^{p-1}<a_{0}(x)
$$

for all $t>0, x \in \bar{\Omega}$. Define $m(x)$ by

$$
m(x)= \begin{cases}f(x, u(x)) / u(x)^{p-1}, & \text { if } x \in \Omega ; \\ a_{0}(x), & \text { if } x \in \partial \Omega,\end{cases}
$$

where $u(x)$ is a positive solution of (3.1). Then $a_{\infty}(x)<m(x)<a_{0}(x)$, hence $m(x) \in L^{\infty}, m^{+} \not \equiv 0$. Since a positive solution $u$ of (3.1) also solves

$$
-\Delta_{p} u=m(x)|u|^{p-2} u \quad \text { in } \Omega, \quad u=0 \text { on } \partial \Omega,
$$

we derive that $\lambda_{1}(m)=1$ from simplicity of positive eigenfunction. Lemma 2.3 further shows that

$$
\lambda_{1}\left(a_{0}\right)<\lambda_{1}(m)=1<\lambda_{1}\left(a_{\infty}\right) .
$$

This completes the proof.

\section{Nonhomogeneous case}

In this section we will deal with the nonhomogeneous problem, that is, $h(x) \not \equiv 0$ in (1.1). We assume that $f$ satisfies (H1) and

$$
\lim _{s \rightarrow \pm \infty} \frac{f(x, s)}{|s|^{p-2} s}=a_{ \pm}(x) \in L^{\infty} .
$$

Thus we can decompose $f(x, u)$ into

$$
f(x, u)=a_{+}(x)|u|^{p-2} u^{+}-a_{-}(x)|u|^{p-2} u^{-}+f_{1}(x, u),
$$

where

$$
\lim _{s \rightarrow \infty} \frac{\max _{|u| \leq s} f_{1}(x, u)}{s^{p-1}}=0 .
$$

We also assume $a_{ \pm}^{+}(x) \not \equiv 0$ so that $\lambda_{1}\left(a_{ \pm}\right)$are well defined. We further restrict ourselves to the case where

$$
\lambda_{1}\left(a_{ \pm}\right)>1
$$


REMARK 4.1. We note that in the case where $a_{ \pm}$are positive constants, (4.2) is equivalent to $a_{ \pm}<\lambda_{1}(1)$. Moreover, in the case $a_{ \pm}(x) \leq \lambda_{1}(1)$ with strict inequalities on subregions in $\Omega$ with positive measure, a condition routinely used in the literature, (4.2) holds. Indeed, (4.2) is slightly more general than that. It is conceivable that $a_{ \pm}>\lambda_{1}(1)$ in some subregions of $\Omega$ but (4.2) remains true.

We first investigate the homogeneous case.

LEMMA 4.1. The problem

$$
-\Delta_{p} u=a_{+}(x)|u|^{p-2} u^{+}-a_{-}(x)|u|^{p-2} u^{-} \quad \text { in } \Omega, \quad u=0 \text { on } \partial \Omega
$$

has only trivial solution.

ProOF. Let $u$ be a solution. Multiplying both sides of (4.3) by $u^{+}$and integrating yield that

$$
\int\left|\nabla u^{+}\right|^{p}=\int a_{+}(x)\left|u^{+}\right|^{p}
$$

On the other hand, for any $u \in W_{0}^{1, p}(\Omega)$ with $\int a_{+}(x)|u|^{p} \neq 0$,

$$
\int|\nabla u|^{p} \geq \lambda_{1}\left(a_{+}\right) \int a_{+}(x)|u|^{p}>\int a_{+}(x)|u|^{p},
$$

by (4.2). It then follows that $u^{+} \equiv 0$. Similarly we derive that $u^{-} \equiv 0$. Thus $u \equiv 0$. The lemma is proved.

Now we define, for $\eta>0$,

$$
\begin{aligned}
F(\eta, v) & =f(x, \eta v) / \eta^{p-1} \\
& =a_{+}|v|^{p-2} v^{+}-a_{-}|v|^{p-2} v^{-}+f(x, \eta v) / \eta^{p-1}
\end{aligned}
$$

and

$$
\Phi(\eta, v)=v-R\left(F(\eta, v)+h / \eta^{p-1}\right),
$$

which maps $L^{\infty}$ into itself.

REMARK 4.2. We note that if $\Phi(\eta, v)=0$ for some $\eta>0$, then $\eta v$ is a solution of (1.1).

LEMMA 4.2. There exist $\eta_{0}>0, M>0$ such that for any $\eta \geq \eta_{0}$ and $\|v\|_{\infty} \geq M$, we have $\Phi(\eta, v) \neq 0$. 
PROOF. Assume, on the contrary, that there exist two sequences $\left\{\eta_{k}\right\}$ and $\left\{v_{k}\right\}$ such that $\eta_{k} \rightarrow \infty,\left\|v_{k}\right\|_{\infty} \rightarrow \infty$, and $\Phi\left(\eta_{k}, v_{k}\right)=0$, that is,

$$
-\Delta_{p} v_{k}=F\left(\eta_{k}, v_{k}\right)+h / \eta_{k}^{p-1} \text {. }
$$

Denote $u_{k}=v_{k} /\left\|v_{k}\right\|_{\infty}$. We then obtain

$$
\begin{aligned}
-\Delta_{p} u_{k} & =\left(F\left(\eta_{k}, v_{k}\right)+h / \eta_{k}^{p-1}\right)\left\|v_{k}\right\|_{\infty}^{-(p-1)} \\
& =a_{+}\left|u_{k}\right|^{p-2} u_{k}^{+}-a_{-}\left|u_{k}\right|^{p-2} u_{k}^{-}+\frac{f_{1}\left(x, \eta_{k} v_{k}\right)+h}{\eta_{k}^{p-1}\left\|v_{k}\right\|_{\infty}^{p-1}} .
\end{aligned}
$$

We thus derive that $\int\left|\nabla u_{k}\right|^{p}$ is bounded. Hence for some $u_{0} \in W_{0}^{1, p}(\Omega)$, there exists a subsequence of $\left\{u_{k}\right\}$, still denoted by $\left\{u_{k}\right\}$, such that $u_{k} \rightarrow u_{0}$ weakly in $W_{0}^{1, p}(\Omega)$ and strongly in $L^{p}$. It follows from compactness of $R$ that $u_{k} \rightarrow u_{0}$ in $L^{\infty}$ and $\left\|u_{0}\right\|_{\infty}=1$. Combining with continuity of $R$ we conclude that

$$
-\Delta_{p} u_{0}=a_{+}(x)\left|u_{0}\right|^{p-2} u_{0}^{+}-a_{-}(x)\left|u_{0}\right|^{p-2} u_{0}^{-} .
$$

Lemma 4.1 then implies that $u_{0} \equiv 0$, which is impossible. Thus $\Phi(\eta, v) \neq 0$ for $\eta$ and $\|v\|_{\infty}$ sufficiently large. The proof of this lemma is complete.

Let $B_{M}$ denote the ball in $L^{\infty}$ with radius $M$ and centered at the origin. It follows from Lemma 4.2 that $\operatorname{deg}\left(\Phi(\eta, v), B_{M}, 0\right)$ is well defined for $\eta \geq \eta_{0}$. Moreover we have

LEMMA 4.3. $\operatorname{deg}\left(\Phi(\eta, v), B_{M}, 0\right) \neq 0$, for $\eta \geq \eta_{0}$.

ProOF. We observe that, by Lemma 4.2

$$
\Psi(t, \eta, v):=v-R\left(a_{+}|v|^{p-2} v^{+}-a_{-}|v|^{p-2} v^{-}+\frac{f_{1}(x, \eta v)+h}{\eta^{p-1}} t^{p-1}\right)
$$

defines a homotopy for $t \in[0,1]$. We then have

$$
\operatorname{deg}\left(\Psi(1, \eta, v), B_{M}, 0\right)=\operatorname{deg}\left(\Psi(0, \eta, v), B_{M}, 0\right) .
$$

On the other hand, since

$$
R\left(a_{+}|v|^{p-2} v^{+}-a_{-}|v|^{p-2} v^{-}\right)
$$

is an odd operator, the Borsuk Theorem implies that

$$
\operatorname{deg}\left(\Phi(\eta, v), B_{M}, 0\right)=\operatorname{deg}\left(\Psi(0, \eta, v), B_{M}, 0\right) \neq 0 .
$$

This ends the proof of the lemma. 
We now state our existence result

THEOREM 4.1. Assume that (4.1) and (4.2) hold with $f_{1}(x, 0)=0$. Then for any $h \in L^{\infty}, h \neq \equiv 0$, problem (1.1) has a nontrivial solution.

PROOF. The existence follows from Lemma 4.3, while $h \not \equiv 0$ implies that the solution cannot be trivial.

REMARK 4.3. In the paper by Boccardo, Drábek and Kučera [3], existence results are obtained for the case (in essence) $a_{ \pm}=\lambda_{1}(1)$.

REMARK 4.4. We note that, if $-\Delta_{p} u$ is replaced by $-\Delta_{p} u+b(x)|u|^{p-2} u$ with $b(x) \in$ $L^{\infty}, b(x) \geq 0$ a.e. in $\Omega$, the same proofs can be carried through and the same results of this paper remain valid.

\section{Acknowledgement}

The author is grateful to the referee for his very detailed and constructive comments on an earlier version of this paper.

\section{References}

[1] A. Anane, "Simplicité et isolation de la première valeur propre du $p$-laplacien avec poids", $C$. $R$. Acad. Sci. Paris Sér. I Math. 305 (1987) 725-728.

[2] J. P. G. Azorero and I. P. Alonso, "Existence and uniqueness for the $p$-Laplacian: nonlinear eigenvalues", Comm. Partial Differential Equations 12 (1987) 1389-1430.

[3] L. Boccardo, P. Drábek and M. Kučera, "Landesman-Lazer conditions for strongly nonlinear boundary value problems", Comment. Math. Univ. Carolin. 30 (1989) 411-427.

[4] H. Brezis and L. Oswald, "Remarks on sublinear elliptic equations", Nonlinear Anal. 10 (1986) 55-64.

[5] D. G. Costa and J. V. A. Gonçalves, "On the existence of positive solutions for a class of nonselfadjoint elliptic boundary value problems", Applicable Anal. 31 (1989) 309-320.

[6] M. Del Pino, M. Elgueta and R. Manasevich, "A homotopic deformation along $p$ of a LeraySchauder degree result and existence for $\left(\left|u^{\prime}\right|^{p-2} u^{\prime}\right)^{\prime}+f(t, u)=0, u(0)=u(t)=0, p>1$ ', $J$. Differential Equations 80 (1989) 1-13.

[7] E. Di Benedetto, " $C^{1+\alpha}$ local regularity of weak solutions of degenerate elliptic equations", Nonlinear Anal. 7 (1983) 827-850.

[8] J. I. Diaz, Nonlinear Partial Differential Equations and Free Boundaries vol. 1: Elliptic Equations, Volume 106 of Research Notes in Mathematics (Pitman Advanced Publishing Program, London, 1985).

[9] D. Gilbarg and N. S. Trudinger, Elliptic Partial Differential Equations of Second Order, 2nd ed. (Springer-Verlag, New York, 1983). 
[10] M. Guedda and L. Veron, "Local and global properties of solutions of quasilinear elliptic equations", J. Differential Equations 76 (1988) 159-189.

[11] P. Hess and T. Kato, "On some linear and nonlinear eigenvalue problems with an indefinite weight function", Comm. Partial Differential Equations 5 (1980) 999-1030.

[12] J. Kazdan and R. J. Kramer, "Invariant criteris for existence of solutions to second order quasilinear equations", Comm. Pure Appl. Anal. 31 (1978) 619-645.

[13] P. Lindqvist, "On the equation $\operatorname{div}\left(|\nabla u|^{\rho-2} \nabla u\right)+\lambda|u|^{\rho-2} u=0 "$, Proc. Amer. Math. Soc. 109 (1990) 157-164.

[14] J. L. Lions, Quelques Méthodes de Résolution des Problèmes aux Limites Non-linéaires (Dunod, Paris, 1969).

[15] M. Otani, "Existence and nonexistence of nontrivial solutions of some nonlinear degenerate elliptic equations", J. Funct. Anal. 76 (1988) 140-159.

[16] M. Otani and T. Teshima, "On the first eigenvalue of some quasilinear elliptic equations", Proc. Japan Acad. Ser. A Math. Sci. 64 (1988) 8-10.

[17] D. H. Sattinger, Topics in Stability Bifurcation Theory, Volume 309 of Lecture Notes in Mathematics (Springer-Verlag, New York, 1973).

[18] J. Serrin, "Local behaviour of solutions of quasilinear equations", Acta Math. 111 (1964) 247-302.

[19] A. Szulkin, "Ljusternik-Schnirelmann theory on $C^{1}$ manifolds", Ann. Inst. H. Poincaré. Anal. Non Linéaire 5 (1988) 119-139.

[20] P. Tolksdorf, "On the Dirichlet problem for quasilinear equations in domains with conical boundary points", Comm. Partial Differential Equations 8 (1983) 773-817. 